\title{
Competition for adhesion between probiotics and human gastrointestinal pathogens in the presence of carbohydrate
}

\author{
Y.-K. Lee* and K.-Y. Puong \\ Department of Microbiology, Faculty of Medicine, National University of Singapore, 5 Science Drive 2, Singapore 117597
}

\begin{abstract}
The adhesion of Lactobacillus rhamnosus GG to human enterocyte-like Caco-2 cells was not inhibited by eight carbohydrates tested, namely $N$-acetyl-glucosamine, galactose, glucose, fructose, fucose, mannose, methyl- $\alpha$-D-mannopyranoside and sucrose. The degree of hydrophobicity predicted the adhesion of $L$. rhamnosus GG to Caco-2 cells. L. rhamnosus GG, however, was able to compete with Escherichia coli and Salmonella spp. of low hydrophobicity and high adhesin-receptor interaction for adhesion to Caco-2 cells. The interference of adhesion of these gastrointestinal (GI) bacteria by L. rhamnosus GG was probably through steric hindrance, and the degree of inhibition was related to the distribution of the adhesin receptors and hydrophobins on the Caco-2 surface. A Carbohydrate Index for Adhesion (CIA) was used to depict the binding property of adhesins on bacteria surfaces. CIA was defined as the sum of the fraction of adhesion in the presence of carbohydrates, with reference to the adhesion measured in the absence of any carbohydrate. The degree of competition for receptor sites between Lactobacillus casei Shirota and GI bacteria is a function of their CIA distance. There were at least two types of adhesins on the surface of $L$. casei Shirota. The study provides a scientific basis for the screening and selection of probiotics that compete with selective groups of pathogens for adhesion to intestinal surfaces. It also provides a model for the characterisation of adhesins and adhesin-receptor interactions.
\end{abstract}

Probiotics: Gastrointestinal pathogens: Competition for adhesion

\section{Introduction}

Adhesion of probiotics to the gastrointestinal (GI) surface is considered a prerequisite for the competitive exclusion of pathogens and for the modulation of local and systemic immunological activities (Salminen et al. 1998; Lee et al. 1999). Specific adhesin-receptor interactions and nonspecific hydrophobic group interactions have been suggested as the major mechanisms for the adhesion of bacteria to GI surfaces (Ofek \& Doyle, 1994). The relative importance of these adhesion mechanisms however is not yet clear.

The stereo-specific adhesin-receptor interaction has been demonstrated to involve carbohydrate moieties on the intestinal surface and carbohydrate-binding adhesins on the bacterial cell surface (Ofek et al. 1978; Adlerberth et al. 1996; Yamamoto et al. 1996). Carbohydrates have been shown to inhibit adhesion of bacteria to the intestinal cell surface (Gusils et al. 1999; Neeser et al. 2000). One may reason that if a suitable soluble carbohydrate could mask the specific adhesin-receptor interaction, the non-specific surface interaction could be studied. Moreover, the carbohydrate inhibition profile of bacterial adhesion to the GI surface would be an indication of the binding characteristics of adhesins.

In this study, the adhesion and competition for adhesion to human intestinal cells (Caco- 2 cell line) by two probiotic Lactobacillus species and eight strains of four GI bacteria, Escherichia coli, Salmonella typhimurium, Salmonella enteritidis and Salmonella bellurup, were studied. It was intended that this study would shed light on the mechanisms of bacterial cell adhesion to the GI surface and the competition between bacteria for adhesion.

\section{Materials and methods Bacterial strains}

The two probiotic lactobacilli studied were Lactobacillus casei strain Shirota (Yakult Singapore Pty. Ltd.) and Lactobacillus rhamnosus strain GG (ATCC 53103). Both bacterial strains have clinically demonstrated probiotic

\footnotetext{
Abbreviations: ATCC, American Type Culture Collection; CIA, Carbohydrate Index for Adhesion; GI, gastrointestinal; NCTC, National Collection of Type Cultures; PBS, Phosphate buffer saline.

*Corresponding author: Dr Y.-K. Lee, fax +65 6 7766872, email micleeyk@nus.edu.sg
} 
properties (Lee et al. 1999). The lactobacilli were cultured in MRS broth (BBL Cockeysville, MD) at $37^{\circ} \mathrm{C}$, in $5 \% \mathrm{v} / \mathrm{v}$ $\mathrm{CO}_{2}$ in air atmosphere, for $18-20 \mathrm{~h}$ before the study.

Escherichia coli O157, Escherichia coli 11775, Salmonella choleraesuis subsp. choleraesuis serotype typhimurium (S. typhimurium) 14028 and Salmonella choleraesuis subsp. choleraesuis serotype enteritidis (S. enteritidis) 13076 were obtained from American Type Culture Collection (ATCC), USA. S. typhimurium E10 (NCTC 8391) was obtained from ), National Collection of Type Cultures (NCTC), UK. The Escherichia coli TG1 (Gibson, 1984) was obtained from the collection of our Department, whereas S. typhimurium $\mathrm{E} 12$ and S. bellurup E23 were faecal isolates provided by the National University Hospital. Bacteria were grown in Luria-Bertani broth (BBL) at $37^{\circ} \mathrm{C}$ for $18-20 \mathrm{~h}$ before use. To radiolabel the bacteria, methyl$1^{\prime}, 2-\left[{ }^{3} \mathrm{H}\right]$ thymidine was added to the medium at a concentration of $10 \mu \mathrm{l} / \mathrm{ml}(117 \mathrm{Ci} / \mathrm{mmol})$. After a period of growth, the bacteria were washed twice with sterile acetate buffer ( $\mathrm{pH} \mathrm{5.0)}$ and then resuspended in the same buffer.

\section{Intestinal cell culture}

Caco- 2 cell cultures were used in the adhesion assay (Fogh et al. 1977). This human colon adenocarcinoma cell line was obtained from ATCC. Cells were cultured in Dulbecco's modified Eagle's minimal essential medium (GIBCOBRL), containing $25 \mathrm{~mm}$-glucose, $20 \% \mathrm{v} / \mathrm{v}$ heat-inactivated fetal calf serum (GIBCO-BRL), and $1 \%$ non-essential amino acids (GIBCO-BRL). Cells were grown at $37^{\circ} \mathrm{C}$, in $5 \% \mathrm{v} / \mathrm{v}$ $\mathrm{CO}_{2}$ in air. For the adhesion assay, monolayers of Caco-2 cells were prepared in 24-well tissue culture dishes (Falcon type 3047 from Becton Dickinson Labware USA) by inoculating $1 \times 10^{5}$ viable cells per well in $1.0 \mathrm{ml}$ of culture medium. The medium was replaced every 2 days.

\section{Carbohydrates tested}

Eight carbohydrates were tested: $N$-acetyl-glucosamine, galactose, glucose, fructose, fucose, mannose, methyl- $\alpha-$ D-mannopyranoside and sucrose. A concentrated solution of the respective carbohydrate was added to a suspension of the bacterium in acetate buffer $(\mathrm{pH} \mathrm{5.0)}$ to give a $25 \mathrm{~mm}$-carbohydrate solution. The adhesion assay was then performed in the presence of the respective carbohydrate.

\section{Carbohydrate Index for Adhesion (CIA)}

The presence of a carbohydrate alters the adhesion properties of bacteria. CIA is an indication of the receptor binding characteristics of bacterial adhesins. The adhesion value without carbohydrate is taken as 1.0 (control) and the adhesion value in the presence of a carbohydrate is taken as a fraction of the control. The CIA is the sum of the fraction of adhesion with reference to the control in the presence of the respective carbohydrate. The CIA distance between a GI bacterium and a lactobacillus is the difference between their CIA values.

\section{Adhesion assay}

Fifteen-days-postconfluent Caco-2 cell monolayers were washed once with $1 \mathrm{ml}$ of sterile acetate buffer ( $\mathrm{pH} 5 \cdot 0$ ) before the adhesion assay. Bacteria at concentrations between $1 \times 10^{8}$ and $1 \times 10^{9} \mathrm{CFU} / \mathrm{ml}$ were added to each well in $1.0 \mathrm{ml}$ (total volume) of acetate buffer $(\mathrm{pH} 5.0$ ) and incubated at $37^{\circ} \mathrm{C}$, in $5 \% \mathrm{v} / \mathrm{v} \mathrm{CO}_{2}$ in air, with gentle rocking. After incubation for $60 \mathrm{~min}$, the monolayers were washed three times with sterile acetate buffer $(\mathrm{pH}$ $5 \cdot 0)$ to remove free bacterial cells. The concentration of adhered bacterial cells was estimated from the radioactivity assayed using liquid scintillation (Ouwehand et al. 2001).

\section{Hydrophobicity measurement}

The hydrophobicity measurement method was a modification of Sweet et al. (1987). Three $\mathrm{ml}$ of the respective bacterial cell suspension in PBS (pH 7.0) was dispensed into $15 \mathrm{ml}$ centrifuge tubes and mixed with $1 \mathrm{ml}$ hexadecane by vortexing vigorously for $30 \mathrm{~s}$. After phase separation (approximately $30 \mathrm{~min}$ ), the aqueous phase was extracted and centrifuged at $3000 \mathrm{rpm}$ for $10 \mathrm{~min}$ in a bench-top centrifuge to remove the remaining hexadecane. The optical density of the aqueous phase was determined at $600 \mathrm{~nm}$. Results were reported as percentage of adherence to hexadecane.

\section{Statistics}

Differences between treatments were examined for the level of significance by the Student's $t$-test after analysis of variance. A $P$-value of $<0.05$ was considered statistically significant.

\section{Results \\ Effect of carbohydrate on adhesion of bacteria to Caco-2 cells}

The percentage change in the adhesion of the two lactobacilli to Caco-2 cells are presented in Figs 1 and 2 . Only $\mathrm{N}$-acetyl-glucosamine among the eight carbohydrates tested inhibited the adhesion of $L$. rhamnosus GG to a significant degree $(P=0.02)$ compared with the control without carbohydrate (Fig. 1). Two of the carbohydrates, namely methyl- $\alpha-D$ mannopyranoside and sucrose, enhanced the adhesion of L. rhamnosus GG $(P<0 \cdot 05)$. The adhesion of $L$. casei Shirota was inhibited by $N$ acetyl-glucosamine, glucose and fucose $(P<0 \cdot 05)$.

The effects of carbohydrates on the adhesion of the other eight strains of GI bacteria are presented in Table 1. Each of the GI bacteria was inhibited by the various carbohydrates to different degrees.

The CIA, the sum of the fraction of adhesion with reference to the control (without carbohydrate) in the presence of the respective carbohydrate, was used to express the cumulative effect of carbohydrates tested on the adhesion of a bacterium. For example, taking adhesion for the control (without carbohydrate) as $1 \cdot 0$, the adhesion of $L$. case $i$ Shirota was 0.68, 0.89, 0.69, 0.93, 0.79, 0.89, 1.0 and 0.98 in the presence of $N$-acetyl-glucosamine, galactose, glucose, 


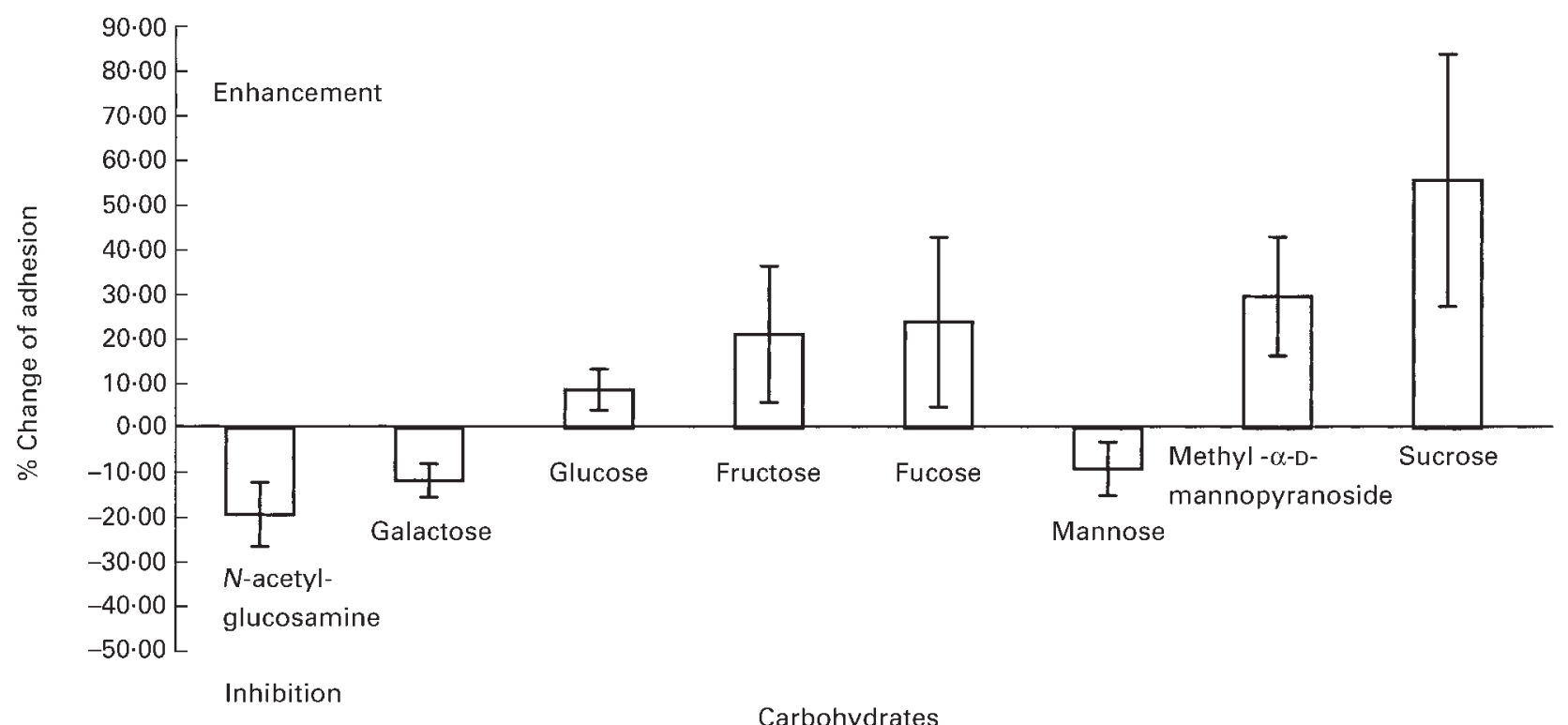

Fig. 1. The effect of carbohydrates on the adhesion of Lactobacillus rhamnosus GG to Caco-2 cells. The vertical bars represent the standard deviation.

fructose, fucose, mannose, methyl- $\alpha-\mathrm{D}-\mathrm{mannopyranoside}$ and sucrose, respectively. The CIA was $0.68+0.89+$ $0.69+0.93+0.79+0.89+1 \cdot 00+0.98=6 \cdot 85$. The CIA values for the ten bacterial strains tested are shown in Table 2. A CIA value close to 8 , such as L. rhamnosus GG (8.99), indicates that the carbohydrates tested had little effect on the adhesion of the bacterium to Caco- 2 cells.

\section{Adhesion of L. casei Shirota to Caco-2 cells in the presence of $\mathrm{N}$-acetyl-glucosamine}

In the adhesion assay for L. casei Shirota, $2 \times 10^{7}$ to $5 \times$ $10^{8}$ cells $/ \mathrm{ml}$ of the lactobacillus were added to a Caco- 2 cell monolayer in the presence or absence of $\mathrm{N}$-acetyl- glucosamine. The plots of the reciprocal of adhered cell concentrations versus the reciprocal of the concentration of cells added are given in Fig. 3. In both the presence and absence of the carbohydrate, a linear relationship was observed. It follows from the equation described below that the intercept on the ordinate gives the value of the reciprocal of the maximum number of bacterial cells adhered to Caco- 2 cells $\left(e_{\mathrm{m}}\right)$. The intercept on the abscissa is $-1 / k_{\mathrm{x}}$, where $k_{\mathrm{x}}$ is the dissociation constant for the adhesion process (Lee et al. 2000).

$$
1 / e_{\mathrm{x}}=1 / e_{\mathrm{m}}+k_{\mathrm{x}} / e_{\mathrm{m}} \cdot x
$$

where $x$ is the concentration of the bacterial culture added, $e_{\mathrm{x}}$ is the concentration of the adhered bacterium.

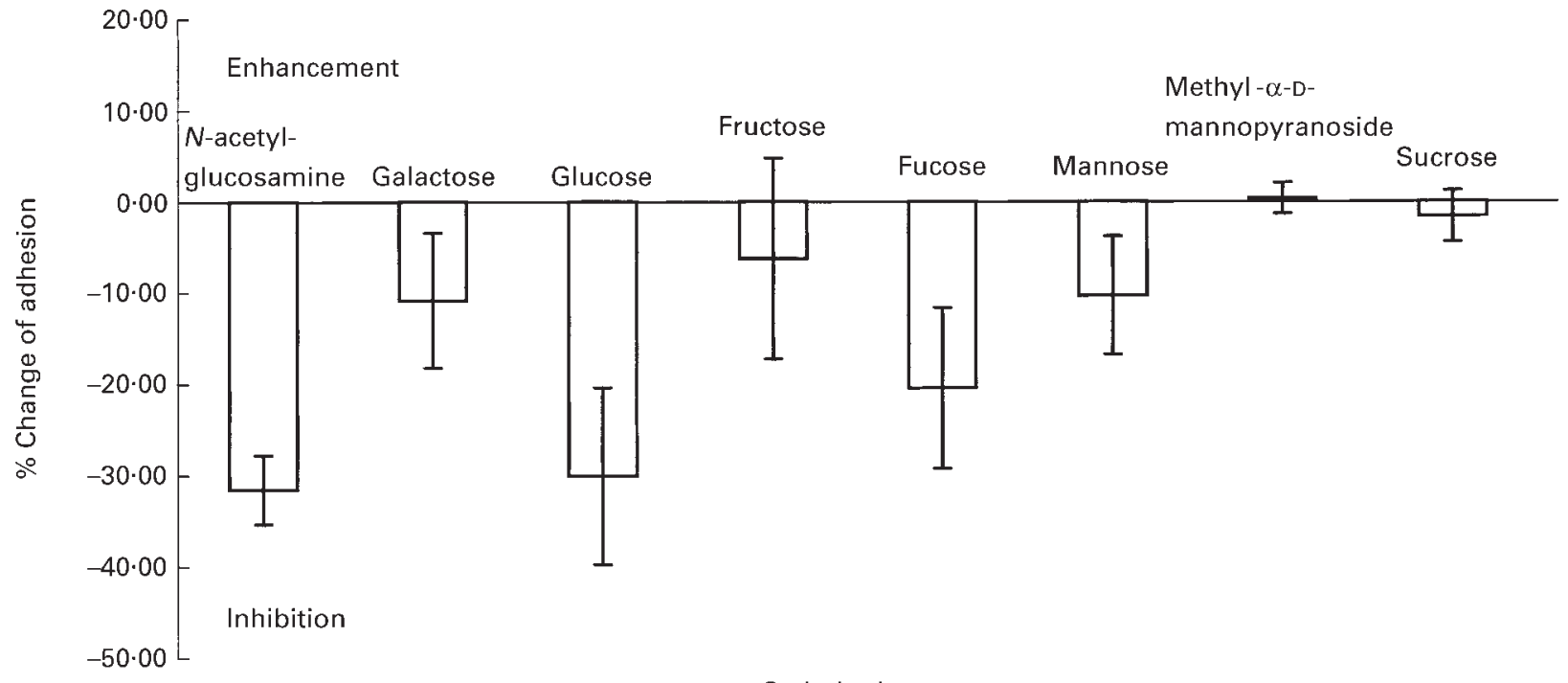

Carbohydrates

Fig. 2. The effect of carbohydrates on the adhesion of Lactobacillus casei Shirota to Caco-2 cells. The vertical bars represent the standard deviation. 
As calculated from the plots shown in Fig. 3 using the above equation, the maximum concentration of adhered L. casei Shirota to Caco-2 cells in the absence of $N$ acetyl-glucosamine was $1.10 \times 10^{7}$ cells/well, whereas the dissociation constant was $6.51 \times 10^{8}$ cells/well. In the presence of $\mathrm{N}$-acetyl-glucosamine, the maximum concentration of adhered $L$. casei Shirota remained unchanged $\left(1.20 \times 10^{7}\right.$ cells/well $)$, whereas the dissociation constant was about three times higher $\left(1.71 \times 10^{9}\right.$ cells $/$ well $)$. The higher dissociation constant in the presence of $\mathrm{N}$-acetylglucosamine implies that $L$. casei Shirota has a lower affinity for adhesion to Caco- 2 cells and adhered cells dissociate more easily in the presence of the carbohydrate.

\section{Hydrophobicity of bacterial cells}

The hydrophobicity of the bacterial cells measured by the PBS method is shown in Table 2. L. rhamnosus GG had the highest hydrophobicity with $73.7 \%$ adhesion to hexadecane, which was followed by $L$. casei Shirota with $66.7 \%$. Among the GI bacteria, E. coli TG1 had the highest hydrophobicity at $63.1 \%$, while S. typhimurium 14028 had the lowest $(5 \cdot 1 \%)$. The other six GI bacteria were around $20 \%$.

\section{Competition for adhesion between L. rhamnosus $G G$ and gastrointestinal bacteria}

Equal concentrations of the respective radiolabelled GI bacterium and L. rhamnosus GG were incubated with Caco-2 cell monolayer for an hour. The reduction in adhesion (competitive exclusion) of the respective GI bacterium by $L$. rhamnosus GG was calculated and plotted against the hydrophobicity of the respective GI bacterium (Fig. 4). No positive correlation between the degree of competitive exclusion (\% reduction of adhesion) and hydrophobicity of the GI bacteria was observed.

\section{Competition for adhesion between L. casei Shirota and gastrointestinal bacteria}

The reduction in the adhesion of the respective GI bacterium in the presence of an equal concentration of L. casei Shirota was determined and plotted against the CIA distance from L. casei Shirota (Fig. 5). The CIA distances were calculated from the difference between the CIA of the respective GI bacterium and that of L. casei Shirota. Two linear relationships were observed. A strong correlation between the degree of competitive exclusion and the CIA distance was found for S. typhimurium 14028, S. bellurup E23 and S. enteritidis 13076. A weaker correlation was found in the case of E. coli TG1, S. typimurium E10, E. coli 11775, E. coli 0157 and S. typimurium E12.

\section{Exclusion of gastrointestinal bacteria by L. casei Shirota}

In this study, L. casei Shirota was allowed to adhere onto Caco-2 cells and then free lactobacillus cells were washed-off. The respective radiolabelled GI bacterium was then added and its adhesion to Caco-2 cells was determined. Figure 6 shows the plot of per cent inhibition of 
Table 2. The Carbohydrate Index for Adhesion of the various bacteria calculated from the data presented in Table 1

\begin{tabular}{|c|c|c|}
\hline Bacterium & Carbohydrate Index for Adhesion & Hydrophobicity (\% adhesion to hexadecane) \\
\hline L. rhamnosus GG & $9 \cdot 0$ & $73.70 \pm 18.76$ \\
\hline L. casei Shirota & 6.9 & $66 \cdot 75 \pm 8.38$ \\
\hline E. coli 0157 & $5 \cdot 4$ & $19.44 \pm 13.28$ \\
\hline E. coli 11775 & $5 \cdot 3$ & $14.23 \pm 11.32$ \\
\hline E.coli TG1 & $6 \cdot 0$ & $63 \cdot 14 \pm 11 \cdot 36$ \\
\hline S. enteritidis 13076 & 6.5 & $22 \cdot 17 \pm 19.95$ \\
\hline S. typhimurium 14028 & $7 \cdot 1$ & $5.08 \pm 19.42$ \\
\hline S. typhimurium E10 & $6 \cdot 1$ & $19.68 \pm 9.71$ \\
\hline S. typhimurium E12 & $4 \cdot 8$ & $19 \cdot 11 \pm 6 \cdot 70$ \\
\hline S. bellurup E23 & $7 \cdot 3$ & $19.62 \pm 5.03$ \\
\hline
\end{tabular}

The hydrophobicity of the various bacterial cells measured by the method of hexadecane partitioning.

adhesion of respective GI bacterium by L. casei Shirota (exclusion) versus the CIA distance from L. casei Shirota. Again, two linear relationships were observed and intercepted at $S$. typhimurium 14028. A strong correlation between the degree of exclusion and CIA distance was apparent for S. typhimurium 14028, S. enteritidis 13076 and $S$. bellurup E23. A weaker correlation was observed for $E$. coli TG1, S. typimurium E10, E. coli 11775, E. coli 0157 and S. typimurium E12.

\section{Displacement of gastrointestinal bacteria by L. casei Shirota}

In this study, the respective radiolabelled GI bacterium was allowed to adhere onto Caco- 2 cells then free GI bacterial cells were washed off. L. casei Shirota was then added and the remaining adhered GI bacteria were determined. Figure 7 shows the plot of per cent inhibition of adhesion of respective GI bacterium in the presence of $L$. casei Shirota (displacement) versus the CIA distance from $L$. casei Shirota. Again, two linear relationships were observed. A strong correlation between the displacement and CIA distance was apparent for S. typhimurium 14028, S. bellurup E23, E. coli TG1, S. enteritidis 13076, E. coli 11775 and $E$. coli 0157. A weaker correlation was observed for $S$. typhimurium E10, and S. typimurium E12.

\section{Discussion}

It is widely suggested that carbohydrate moieties on the intestinal surface and carbohydrate-binding adhesins on the bacterial cell surface are involved in the stereospecific adhesin-receptor interaction leading to the adhesion of bacterial cells to the intestinal surface (Ofek et al. 1978; Adlerberth et al. 1996; Yamamoto et al. 1996). The eight carbohydrates tested in this study inhibited the adhesion of $L$. casei Shirota, the eight strains of E. coli and the Salmonella spp. to the Caco-2 intestinal cell line, but to varying degrees. Interestingly, the adhesion of L. rhamnosus GG to Caco-2 cells was affected by only one of the carbohydrates tested. This may suggest that the carbohydrate-mediated adhesin-receptor interaction is not the major mechanism involved in the adhesion of L. rhamnosus GG to Caco-2 cells. L. rhamnosus GG cell surface showed high hydrophobicity. The non-specific hydrophobic surface interaction may be the main adhesion mechanism for L. rhamnosus GG. Among the eight strains of E. coli and Salmonella spp. tested, only E. coli TG1 showed relatively high hydrophobicity, whereas the surface hydrophobicity of the other seven strains was low. However, L. rhamnosus GG was found to compete with seven strains of E. coli and Salmonella spp. and enhance the adhesion of $E$. coli 0157 . As there is no obvious direct correlation between the degree of competition for adhesion

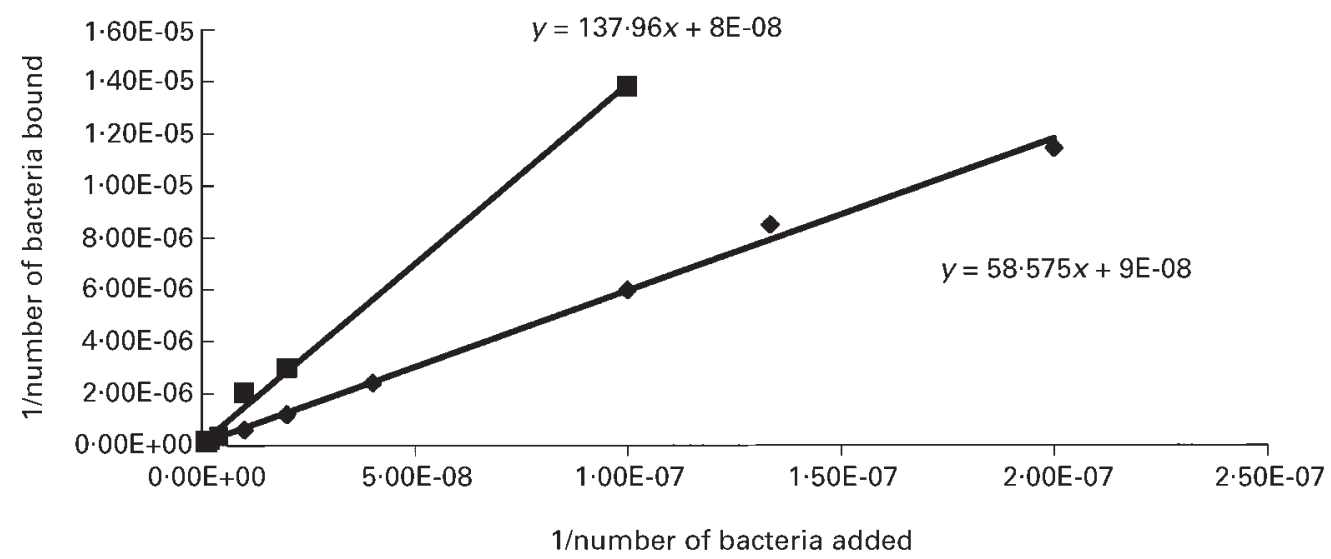

Fig. 3. The plots of $1 /$ number of $L$. casei Shirota bound to Caco-2 cells versus $1 /$ number of $L$. casei Shirota added to Caco-2 cultures, in the presence and absence of $N$-acetyl-glucosamine. ( $), 1$ /number of bacteria bound (without $N$-acetyl-glucosamine); $(\mathbf{\square}), 1 /$ number of bacteria bound (with $N$-acetyl-glycosamine). 


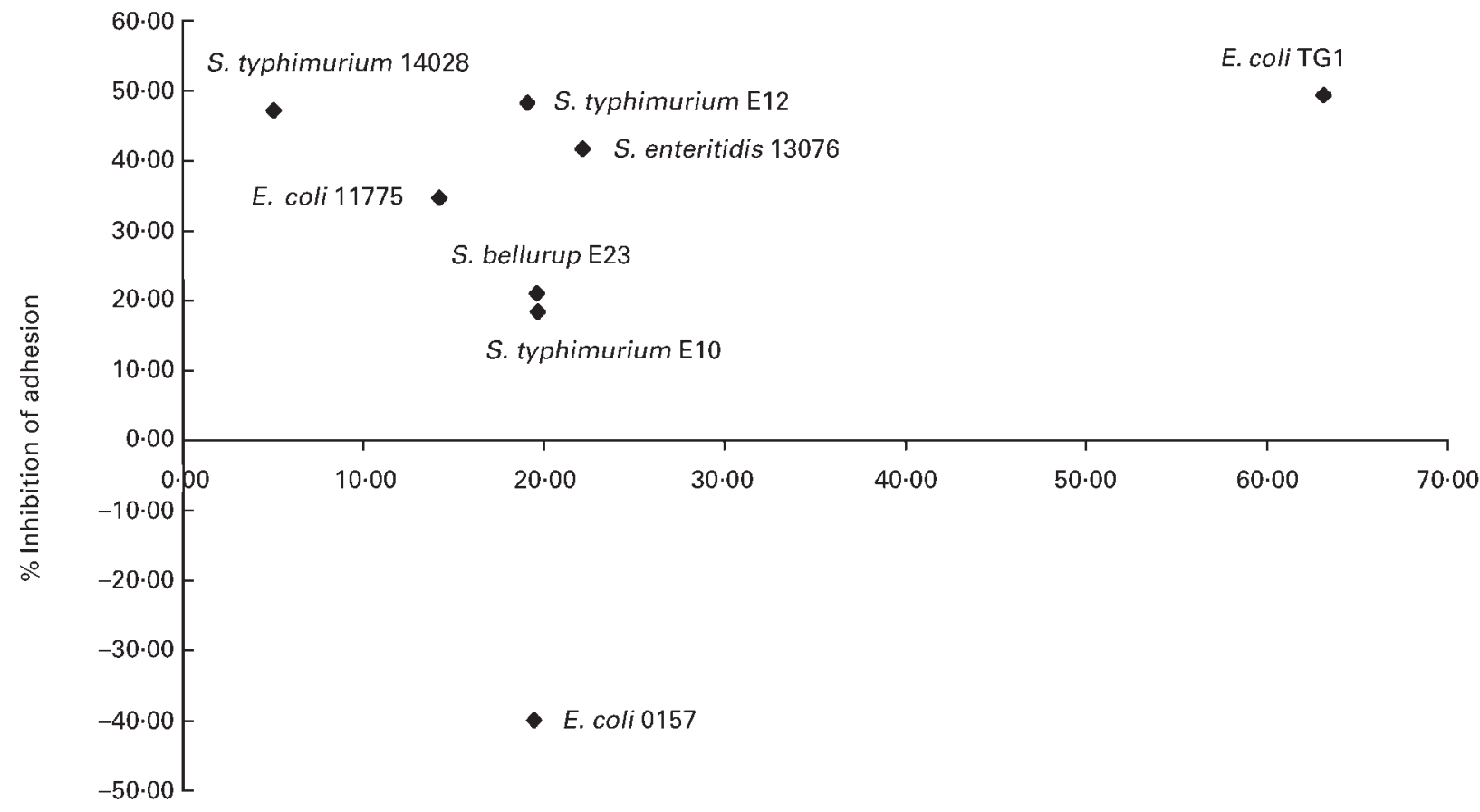

Hydrophobicity

Fig. 4. Correlation between the per cent inhibition of adhesion of GI bacteria (E. coli and Salmonella spp.) to Caco-2 cells by L. rhamnosus $\mathrm{GG}$ and the hydrophobicity of the GI bacteria.

and hydrophobicity of the bacterial cells, the inhibition of adhesion of E. coli and Salmonella spp. to Caco-2 cells by L. rhamnosus GG was probably due to steric hindrance (Chauviere et al. 1992), and the degree of inhibition was related to the relative position of the hydrophobic surface and adhesin receptors. The hydrophobic surface and adhesins on the surface of $E$. coli 0157 were probably a distance apart, and the enhancement of adhesion by L. rhamnosus GG may be due to adhesion of E. coli 0157 cells on L. rhamnosus GG (Ouwehand et al. 2000).
L. rhamnosus GG was reported to increase the adhesion of $S$. typhimurium on human intestinal glycoproteins (Tuomola et al. 1999).

If $\mathrm{N}$-acetyl-glucosamine could mask the major adhesins on the surface of L. casei Shirota, the adhesion measured in the presence of the carbohydrate would represent the hydrophobic interaction between L. casei Shirota and Caco-2 cells. Interestingly, the calculated maximum concentrations of adhered $L$. casei Shirota to Caco-2 cells in the presence and absence of $\mathrm{N}$-acetyl-glucosamine were

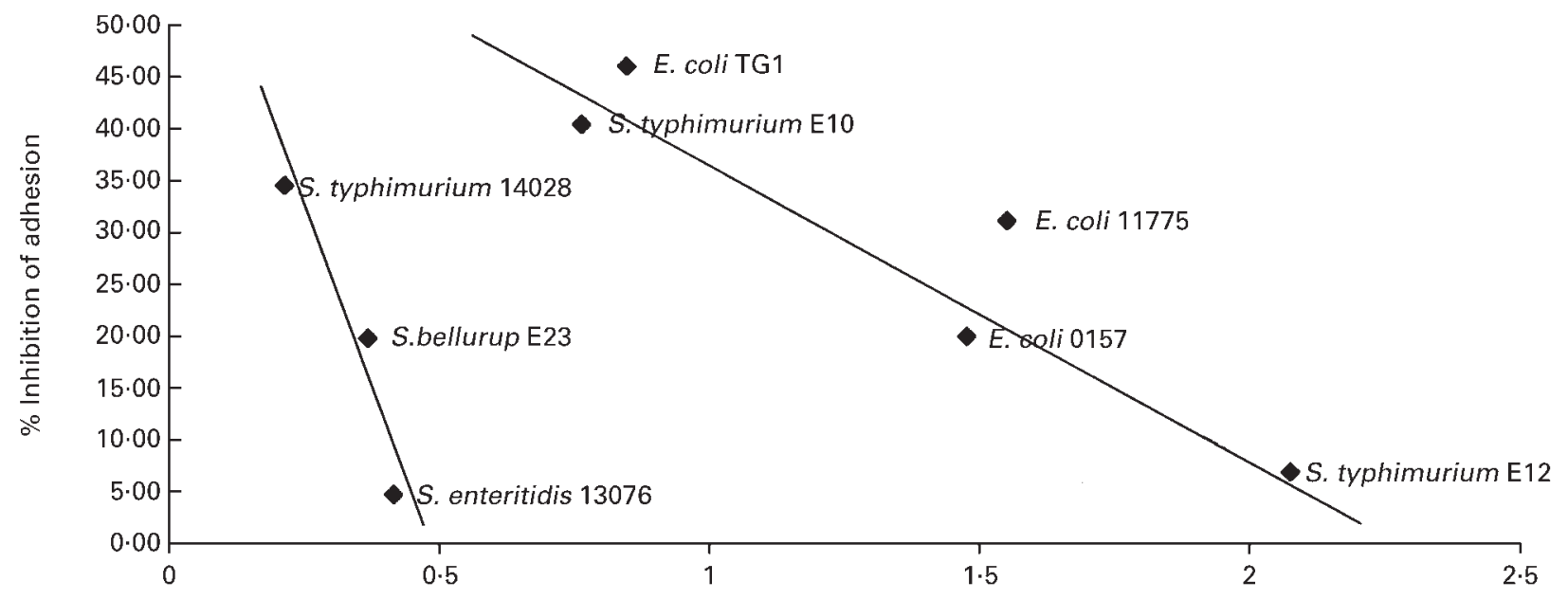

CIA distance from L. casei Shirota

Fig. 5. Correlation between the competitive inhibition of adhesion (per cent inhibition of adhesion compared to adhesion measured in the absence of L. casei Shirota) of GI bacteria (E. coli and Salmonella spp.) to Caco-2 cells by L. casei Shirota and their Carbohydrate Index for Adhesion (CIA) distances. 


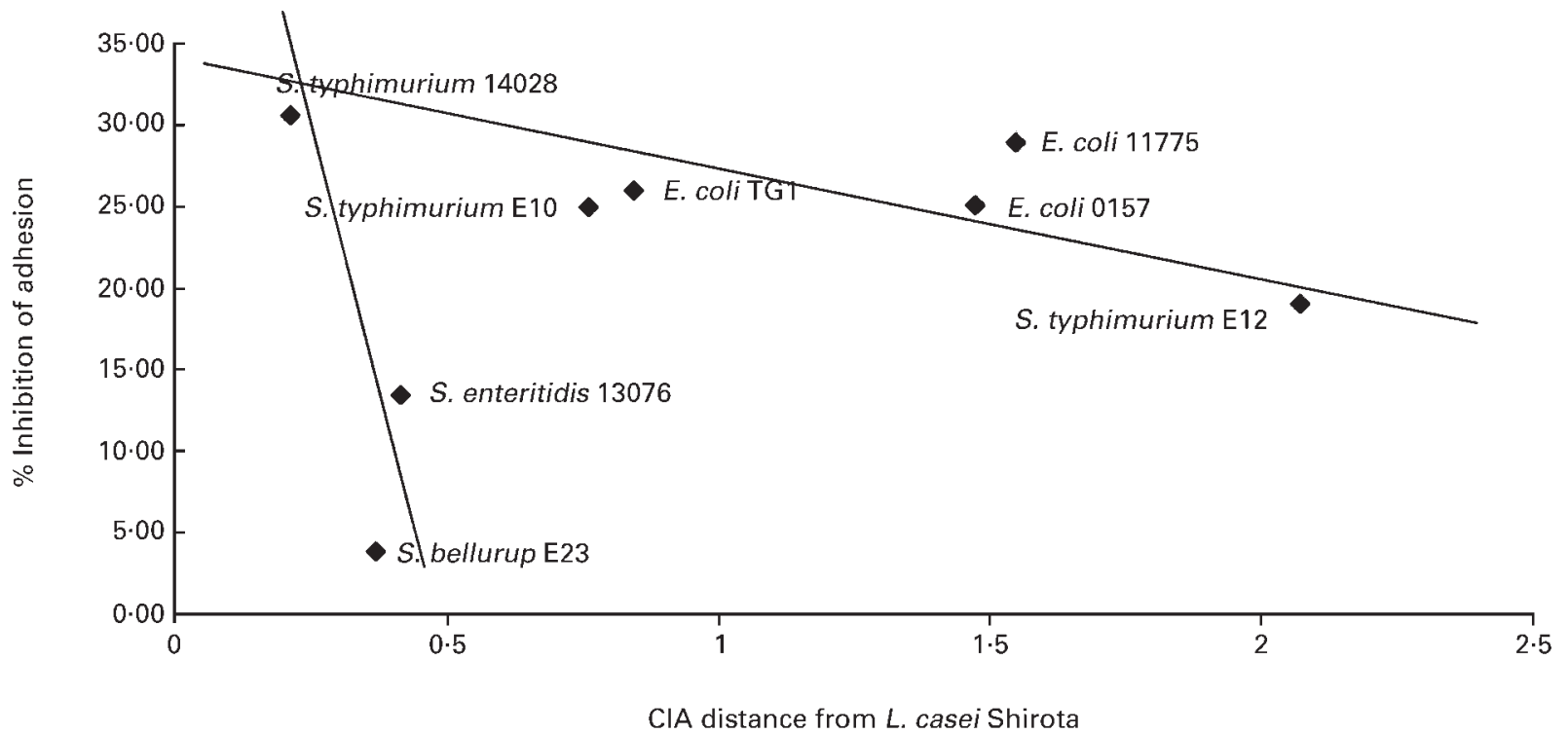

Fig. 6. Correlation between the exclusion (per cent inhibition of adhesion compared to adhesion measured in the absence of $L$. casei Shirota) of GI bacteria (E. coli and Salmonella spp.) from Caco-2 cells by L. casei Shirota and their Carbohydrate Index for Adhesion (CIA) distances.

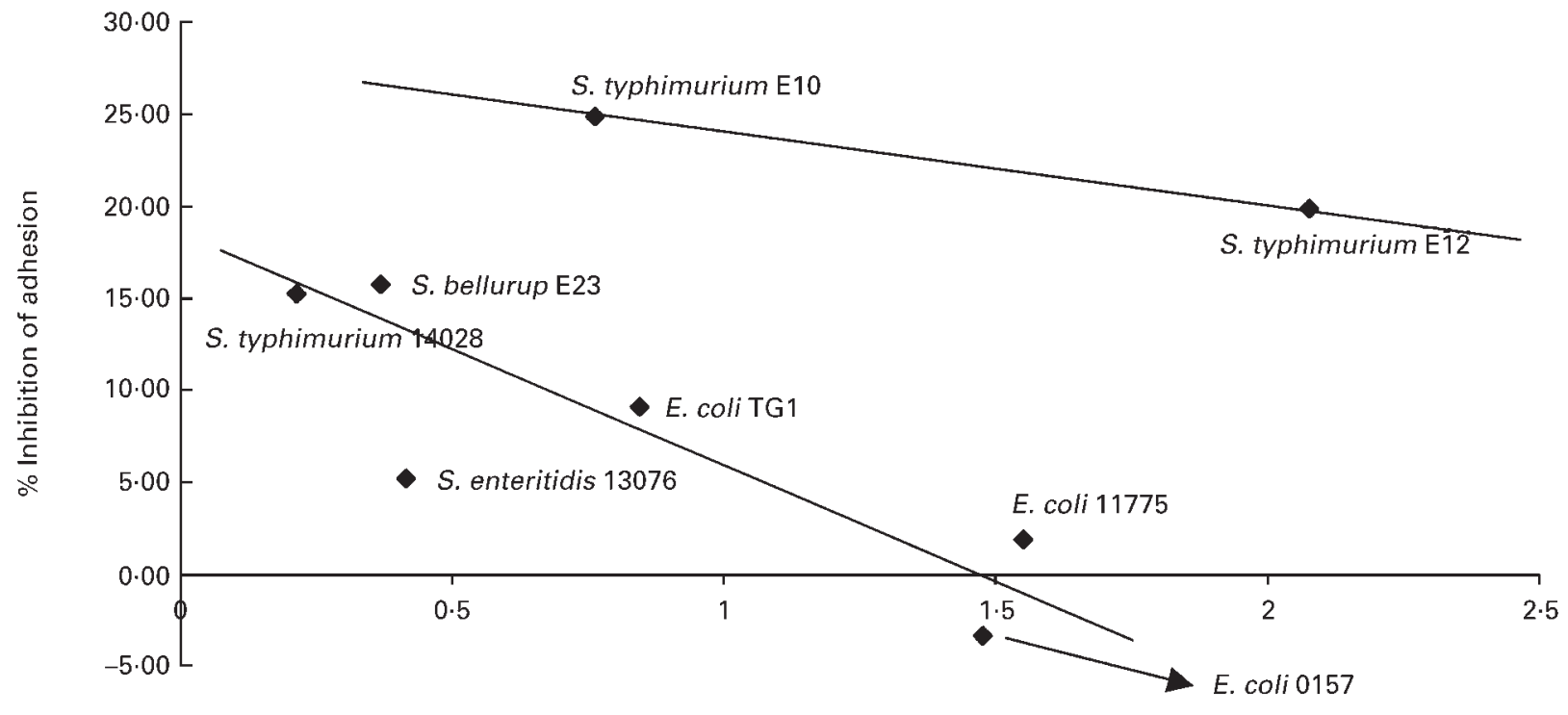

CIA distance from L. casei Shirota

Fig. 7. Correlation between the displacement (per cent inhibition of adhesion compared to adhesion measured in the absence of $L$. casei Shirota) of GI bacteria (E. coli and Salmonella spp.) to Caco-2 cells by L. casei Shirota and their Carbohydrate Index for Adhesion (CIA) distance.

identical. This suggests that the adhesins and the hydrophobic surfaces on L. casei Shirota are in close proximity and have the same number of adhesion sites. For example, the adhesin is located at the tip of a fimbriae, whereas the stalk of the fimbriae is hydrophobic. As expected, the affinity of the adhesin for the Caco-2 receptor was about 2.6 times that of the hydrophobic surface interaction. The hydrophobic surfaces may serve to bring together the surfaces, but it is the adhesin-receptor interaction that plays the major role in the binding of L. casei Shirota and Caco-2 cells.

Based on the carbohydrate inhibition of adhesion profile, the adhesins on L. casei Shirota and the eight strains of E. coli and Salmonella spp. are probably not identical.
The concept of CIA is introduced in the work to depict the receptor binding characteristic of the adhesins on bacterial surface. Two different bacteria with identical CIA would imply identical adhesins and the distance between their CIA values is zero. The greater the degree of difference between the CIA values, the greater would be the differences between the adhesins on the bacteria. In the competition study, when the degrees of inhibition of adhesion of the eight strains of E. coli and Salmonella spp. by $L$. casei Shirota were plotted against the distance between their CIA, two linear relationships were observed. This suggests that there are possibly two distinct types of adhesins on the surface of $L$. casei Shirota. One is closely related to the adhesins possessed by S. typhimurium 14028, 
S. bellurup E23 and S. enteritidis 13076, while the other resembles that of $E$. coli TG1, 11775, 0157 and S. typhimurum E10, E12. Two types of carbohydrate-binding adhesins were found on the surface of L. johnsonii La1 (Neeser et al. 2000), one for O-linked oligomannosides and the other for the gangliotri- and gangliotetra-osylcer amides. The difference between the CIA values determined the degree of competitive exclusion of a pathogen by L. casei Shirota for adhesion to Caco-2 cells. This approach provides a scientific basis for the screening and characterization of probiotic lactobacilli for competitive exclusion of a specific pathogen or groups of pathogens.

The same trend was observed in the exclusion of pathogens by $L$. casei Shirota: the same two groups of $E$. coli and Salmonella spp. showed two distinct linear relationships between the degrees of exclusion of the pathogens by $L$. casei Shirota for adhesion of Caco-2 cells.

The displacement of the eight strains of E. coli and Salmonella spp. by L. casei Shirota showed two linear relationships. However, the $E$ coli strains had joined the group of S. typhimurium 14028, S. enteritidis 13076 and $S$. bellurup E23, while $S$. typhimurium E10 and E12 remained separated. A second mechanism may have been involved in the displacement of E. coli by L. casei Shirota from the receptors on Caco- 2 cells.

This study provides some direction for future molecular and genetic studies of adhesins on bacteria and receptors on intestinal surfaces. The study also provides a rational approach for the screening and selection of probiotics with desirable properties.

\section{References}

Adlerberth I, Ahrne S, Johansson M-L, Molin G, Hanson LA \& Wold AE (1996) A monose-specific adherence mechanism in Lactobacillus plantarum conferring binding to the human colonic cell line HT-29. Applied and Environmental Microbiology 62, 2244-2251.

Chauviere G, Coconnier MH, Kerneis S, Darfeuille-Michaud A, Joly B \& Servin AL (1992) Competitive exclusion of diarrheagenic Escherichia coli (ETEC) from human enterocyte-like Caco-2 cells by heat-killed Lactobacillus. FEMS Microbiology Letter 70, 213-217.

Fogh J, Fogh JM \& Orfeo T (1977) One hundred and twenty- seven cultured human tumour cell lines producing tumours in nude mice. Journal of National Cancer Institute 59, 221-226.

Gibson TJ (1984) Studies on the Epstein-Bar virus genome. PhD Thesis, Cambridge University, UK.

Gusils C, Gonzalez SN \& Oliver G (1999) Some probiotic properties of chicken lactobacilli. Canadian Journal of Microbiology 45, 981-987.

Lee YK, Lim CY, Teng WL, Ouwehand AC, Tuomola EM \& Salminen S (2000) Quantitative approach in the study of adhesion of lactic acid bacteria to intestinal cells and their competition with enterobacteria. Applied and Environmental Microbiology 66, 3692-3697.

Lee YK, Nomoto K, Salminen S \& Gorbach SJ (1999) Handbook of Probiotics. New York: John Wiley \& Sons.

Neeser J-R, Granato D, Rouvet M, Servin A, Teneberg S \& Karlsson K-A (2000) Lactobacillus johnsonii La1 shares carbohydrate-binding specificities with several enteropathogenic bacteria. Glycobiology 10, 1193-1199.

Ofek I, Beachey EH \& Sharon N (1978) Surface sugars of animal cells as determinants of recognition in bacterial adherence. Trends in Biochemical Science 3, 159-160.

Ofek I \& Doyle RJ (1994) Bacterial Adhesion to Cells and Tissues. New York: Chapman \& Hall.

Ouwehand AC, Isolauri E, Kirjavainen PV, Tolkko S \& Salminen SJ (2000) The mucus binding of Bifidobacterium lactis Bb12 is enhanced in the presence of Lactobacillus GG and Lact. delbruechii subsp. bulgaricus. Letters in Applied Microbiology 30, 10-13.

Ouwehand AC, Tuomola EM, Lee YK \& Salminen S (2001) Microbial interaction to intestinal mucosal models. In Methods in Enzymology, Vol. 337, pp. 200-212 [RJ Doyle, editor]. New York: Academic Press.

Salminen S, Bouley MC, Boutron-Rualt MC, Cummings J, Franck A, Gibson G, Isolauri E, Moreau MC, Roberfroid M \& Rowland I (1998) Functional food science and gastrointestinal physiology and function. British Journal of Nutrition 80, Suppl. 1, S147-S171.

Sweet SP, MacFarlane TW \& Samarayanake LP (1987) Determination of the cell hydrophobicity of oral bacteria using a modified hydrocarbon adherence method. FEMS Microbiology Letters 48, 135-143.

Tuomola EM, Ouwehand AC \& Salminen SJ (1999) The effect of probiotic bacteria on the adhesion of pathogens to human intestinal mucus. FEMS Immunology and Medical Microbiology 26, 137-142.

Yamamoto K, Miwa T, Taniguchi H, Nagano T, Shimamura K, Tanaka T \& Kumagi H (1996) Binding specificity of Lactobacillus to glycolopids. Biochemical and Biophysical Research Communications 228, 148-152. 